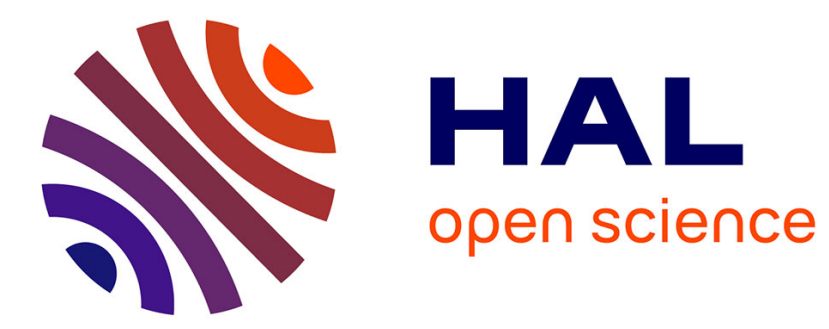

\title{
Des langues chez Volodine: un drame de la survie
}

Frédérik Detue

\section{To cite this version:}

Frédérik Detue. Des langues chez Volodine: un drame de la survie. Littérature, 2008, 151 (3), pp.75-89. 10.3917/litt.151.0075 . hal-02613979

\section{HAL Id: hal-02613979 \\ https://hal.science/hal-02613979}

Submitted on 8 Jun 2020

HAL is a multi-disciplinary open access archive for the deposit and dissemination of scientific research documents, whether they are published or not. The documents may come from teaching and research institutions in France or abroad, or from public or private research centers.
L'archive ouverte pluridisciplinaire HAL, est destinée au dépôt et à la diffusion de documents scientifiques de niveau recherche, publiés ou non, émanant des établissements d'enseignement et de recherche français ou étrangers, des laboratoires publics ou privés. 
Frédérik Detue

\title{
Des langues chez Volodine : un drame de la survie
}

\author{
Durant son agonie, Lutz Bassmann souhaitait uniquement remuer \\ les braises dont il avait la garde, et ne pas être absorbé trop vite, \\ avec elles, par le néant. \\ Antoine Volodine, Le Post-exotisme en dix leçons, leçon onze ${ }^{1}$
}

La fiction, dans les textes d'Antoine Volodine, invite à considérer le rôle du signataire comme mineur : ce sont les personnages qui sont les narrateurs - dits post-exotiques - des récits que nous lisons, et quant à Volodine, il n'est qu'un prête-nom, un prête-voix, un porteparole, ou encore un traducteur.

Prenant le parti de cette fiction, je voudrais ici étudier ce qu'implique le fait de penser l'ensemble du corpus volodinien comme une œuvre de traduction, en m'appuyant pour commencer sur ce qu'en a dit l'écrivain dans une conférence, en 2001, intitulée «Ecrire en français une littérature étrangère » :

[Mes romans] forment un objet littéraire publié en langue française, mais pensé en une langue extérieure au français, indistincte quant à sa nationalité. Une langue non rattachée à une aire géographique déterminée, et clairement « étrangère », puisqu'elle ne véhicule pas la culture et les traditions du monde français ou francophone. [...] la langue est [...] un immense territoire international. C'est un territoire indifférencié qui a reçu les traductions de très nombreuses autres langues du monde, et qui non seulement les a reçues, mais les a adoptées, les a portées, les a intégrées. [...] j'ai eu pour souci d'écrire mes livres dans cette langue de traduction. Au niveau du vocabulaire et de la syntaxe, avec toute la souplesse, la richesse, le génie de la langue française, mais pour servir une culture qui soit étrangère aux habitudes de la société française et de l'univers francophone.

La langue de mes livres porte, avant tout, la culture de mes personnages, des écrivains-chamanes que je mets en scène et des lecteurs que j'imagine. Elle véhicule leur culture subversive, cosmopolite et marginale, une culture de rêveurs et de combattants politiques qui ont perdu toutes leurs batailles et qui ont encore le courage de parler, alors qu'ils ont aussi perdu la bataille contre le silence. [...] A quoi ressemble le langage dans lequel ils s'expriment? A une langue variée et parfois pauvre, parfois mutilée ou, au contraire, luxuriante et baroque. Leur langue n'est pas une langue nationale, mais la langue trans-nationale des conteurs d'histoires, des exclus, des prisonniers, des fous et des morts. Je suis ici porte-parole de leurs voix. Dans mes livres, je traduis en français les fictions qu'ils produisent pour protester contre le réel, pour saboter le réel ou pour transformer le réel. ${ }^{2}$

\footnotetext{
${ }^{1}$ VOLODINE, Antoine. Le Post-exotisme en dix leçons, leçon onze. Paris : Gallimard, 1998, p. 17. Référence abrégée : $P E$.

${ }^{2}$ VOLODINE, A. Ecrire en français une littérature étrangère. Chaö̈ [en ligne] : International, automne-hiver 2002, n 6. Format PDF, p. 53, 54-55. Disponible sur : http://www.chaoid.com/pdf/chaoid_6.zip. (Consulté le 05.08.04.) La référence à cette deuxième note sera reprise par un 2 en exposant chaque fois je citerai de nouveau un extrait du texte de Volodine.
} 
Nous comprenons que, suivant le dispositif fictionnel mis en place par Volodine, les œuvres du post-exotisme constituent une histoire littéraire à part, une "culture subversive, cosmopolite et marginale $»^{2}$. La parole de l'écrivain, mais également le texte des œuvres, font apparaître que le sort réservé à cette littérature est de l'ordre du rejet, de l'exclusion, et il est patent que cette exclusion est politique. Qu'elle ait été une «littérature dite des filières (alias littérature des réseaux, alias littérature des poubelles)»" ou qu'elle soit devenue une «littérature carcérale » $(P E, 19)$, demeure l'image d'une littérature qui survit "dans les souterrains de la culture », en marge d'une «littérature officielle, humaniste » (LDM, 147) avec laquelle elle ne dialogue pas ( $c f . P E, 33),-$ l'image d'une littérature dominée, mal reçue, dont l'existence même est fortement menacée.

Non pas qu'on puisse parler à son propos de littérature engagée. Mais les personnages $\mathrm{du}$ post-exotisme qui la produisent sont des «combattants politiques qui ont perdu toutes leurs batailles $»^{2}$; ils sont les derniers révolutionnaires égalitaristes, et leur situation de prise de parole est d'une extrême violence, en prison (dans un quartier de haute sécurité), en camp, en asile ou en exil. Chez Volodine comme chez Zamiatine, deux lois sont opposées : la loi de la révolution et la loi de l'entropie ${ }^{4}$. Volodine synthétisant l'histoire catastrophique du XXe siècle, la loi de l'entropie est au pouvoir, le monde est totalement contre-révolutionnaire. Ainsi la domination que subissent les personnages est-elle toujours de nature totalitaire : c'est un pouvoir dont l'institution centrale est un système de détention pour hommes et femmes de trop; et ils sont ces hommes et femmes de trop: des êtres qu'on soustrait au monde proprement dit, et qu'on fait disparaitre dans le monde concentrationnaire comme s'ils n'avaient jamais existé. Or, si ces vaincus n'ont d'autre perspective, devant eux, que la folie et la mort, s'ils sont déjà dans un état intermédiaire entre la vie et la mort, entre l'humain et le non-humain, ils trouvent néanmoins la force de produire une littérature, et celle-ci est encore faite «pour protester contre le réel, pour saboter le réel ou pour transformer le réel $»^{2}$. Pour eux, engagés écrivains plutôt que l'inverse, écrire est un prolongement de leur action militante, la dernière forme d'action qui leur reste. Tandis qu'on entend anéantir leur culture de la révolution dans le monde concentrationnaire, ils font œuvre pour sauver cette culture du néant.

La question de la langue surgit alors comme un paradoxe, si l'on observe quelle est l'invention de Volodine. Bien qu'il s'agisse dans le post-exotisme de culture de la révolution,

\footnotetext{
${ }^{3}$ VOLODINE, A. Lisbonne, dernière marge. Paris : Editions de Minuit, 1990, p. 147. Référence abrégée : LDM.

${ }^{4}$ Cf. ZAMIATINE, Evgueni. Le Métier littéraire : portraits, études et manifestes. Trad. du russe par F. Monat. Lausanne : L'Age d'Homme, 1990. Coll. Slavica. Littérature, révolution et entropie, p. 150-155.
} 
et qu'il n'existe aucune langue de la révolution attestée, il n'est tout de même pas vain d'y lier culture et langue ; car c'est justement une utopie dans l'œuvre de Volodine que l'imagination d'une telle langue: nous découvrirons que l'écrivain ne parle pas d'autre chose quand il qualifie la langue de ses narrateurs post-exotiques de langue «indistincte quant à sa nationalité », de "langue trans-nationale ${ }^{2}$. Il s'agira de considérer en quoi cette langue de la révolution est une arme de combat révolutionnaire, mais surtout de se demander dans quelle mesure elle peut être encore une telle arme, alors que la révolution se meurt et que ses rares locuteurs survivants sont en voie d'extinction. Telle peut être une formulation du paradoxe : certes, l'enjeu de la prise de parole dans cette langue est bel et bien de lutter contre l'anéantissement d'une culture, mais le drame de cette parole, c'est que se communique dans le même temps cet anéantissement lui-même, à l'œuvre de façon performative. Et si la traduction de Volodine est alors ce qui reste de ce drame, elle n'a pas vocation cependant à le dénouer : seulement - mais c'est beaucoup - à en témoigner.

Dire d'une langue qu'elle est transnationale, c'est en faire une langue de passage, une langue entre les langues, qui manifeste leur parenté entre elles, et qui met un cap vers elles. La langue des narrateurs post-exotiques apparaît ainsi théoriquement comme une langue grosse de toutes les langues du monde, qui les porte en elle, comme en germe, à un stade prénatal ; pure traductibilité, c'est une langue qui appelle la traduction dans toutes les langues (et, par exemple, celle de Volodine en français).

Mais il faut bien voir que ce cap qu'elle met vers les langues est destructeur, cependant. L'opposition de Zamiatine entre révolution et entropie, qui fonctionne comme un paradigme dans l'œuvre de Volodine, opère entre la langue transnationale post-exotique et les langues nationales. "Pourpre, ardente, mortelle est la loi de la révolution ${ }^{5}$, et telle est la langue de la révolution : une langue de feu, éruptive, une langue-mouvement que rien n'est venu figer dans une identité, et qui s'élance vers les langues entropiques exactement comme la lave éruptive dévaste la croûte solide et beaucoup plus récente des vieilles explosions sédimentées.

Nul mieux que Paul Celan, peut-être, n'a formulé ce qu'il en est de l'entropie dans les langues :

\footnotetext{
${ }^{5}$ Ibid., p. 151.
} 
Accessible, proche et non perdu demeura au milieu de toutes les pertes seulement ceci : la langue.

Elle, la langue, demeura non perdue, oui, malgré tout. Mais elle devait à présent traverser ses propres absences de réponse, traverser un terrible mutisme, traverser les mille ténèbres de paroles porteuses de mort. Elle les traversa et ne céda aucun mot à ce qui arriva ; mais cela même qui arrivait, elle le traversa. Le traversa et put revenir au jour, «enrichie » de tout cela.

Dans cette langue j'ai essayé durant ces années et les années qui suivirent d'écrire des poèmes $[\ldots]{ }^{6}$

Voilà ce qui arrive aux langues, ici l'allemand : une langue dont Victor Klemperer a consacré sa survie, entre 1933 et 1945, à noter scrupuleusement comme elle est devenue le "dépôt de la catastrophe " ${ }^{7}$, et comme telle, complice et meurtrière, corrompant la parole et dévorant la pensée aussi bien des victimes que des bourreaux. Alors, pour Paul Celan, être un poète juif de langue allemande après la Shoah, c'est écrire dans l'allemand contre l'allemand, dans une langue qui "se méfie du "beau", [...] une langue "plus grise" », contre «ces "harmonies" qui en compagnie et au voisinage de l'horreur continuèrent plus ou moins tranquillement à se faire entendre ${ }^{8}$, contre cette langue-de-mort qui continue d'empoisonner la parole et la pensée allemandes après la défaite du IIIe Reich. Et quant aux personnages du post-exotisme, qui se mettent à écrire tandis qu'on tente, à l'échelle mondiale, d'accomplir le meurtre de la révolution, devenir un collectif de révolutionnaires écrivains de langue transnationale, c'est aussi écrire dans une contre-langue, mais en l'occurrence, dans une langue contre toutes les langues $d u$ monde, parce qu'elles sont toutes également porteuses de cette mort catastrophique de la révolution.

S'attaquer aux langues avec une langue en révolution, c'est une lutte à rebours de leur évolution, de leur entropie ; et c'est pourquoi cela conduit à retraverser, en sens inverse, tout ce dont elles se sont «enrichies» («[leurs] propres absences de réponse, [...] un terrible mutisme, [...] les mille ténèbres de paroles porteuses de mort») : ainsi Paul Celan revient-il incessamment sur la catastrophe du génocide nazi; ainsi les narrateurs post-exotiques reviennent-ils incessamment sur la catastrophe que représente l'échec du projet révolutionnaire au XXe siècle. Creuser dans ses souvenirs, ratisser ses terres mémorielles comme le font les narrateurs post-exotiques de livre en livre, c'est, au-delà même de l'oubli, mettre un cap vers les langues où cette mémoire s'est déposée pour la retrouver, et c'est, avec sa contre-langue, creuser dans ces langues entropiques jusqu'à trouver le vide dedans, faire

\footnotetext{
${ }^{6}$ CELAN, Paul. Le Méridien \& autres proses. Trad. de l'allemand par Jean Launay. Paris : Le Seuil, 2002. Coll. La Librairie du XXIe siècle. Allocution de Brême, p. 56-57.

${ }^{7}$ BROSSAT, Alain. Postface : Résistance dans la langue. KLEMPERER, Victor. LTI, la langue du IIIe Reich : Carnets d'un philologue. Trad. de l'allemand par Elisabeth Guillot. Paris : Pocket, 2003. Coll. Agora, p. 368.

${ }^{8}$ CELAN, P. Op. cit. Réponse à une enquête de la librairie Flinker, Paris (1958), p. 31-32.
} 
trou dans les langues, et leur faire perdre le sens, au grand dam de l'ennemi contrerévolutionnaire : "On élabore une proclamation solennelle, dans une langue qui paraît être la même que celle des juges, mais que les juges écoutent avec consternation ou ennui, car ils sont incapables d'en percer le sens » (PE, 11-12).

«A quoi ressemble le langage dans lequel [mes personnages] s'expriment? A une langue variée et parfois pauvre, parfois mutilée ou, au contraire, luxuriante et baroque. " ${ }^{2}$, souligne Volodine. Leur contre-langue est une langue de l'excès, de la démesure ; et faire la révolution dans les langues entropiques comme elle s'y destine, c'est très exactement ce que Gilles Deleuze et Félix Guattari ont appelé minorer les langues, dans Kafka: Pour une littérature mineure: ou "procéder par exubérance et surdétermination » (comme dans l'usage de l'anglais chez Joyce), ou «[procéder] à force de sécheresse et de sobriété, de pauvreté voulue » (comme dans l'usage de l'anglais et du français chez Beckett, ou dans celui de l'allemand chez Kafka) ${ }^{9}$. On conçoit ici la tâche qui incombe au traducteur Volodine par rapport au français ; c'est comme si les œuvres post-exotiques mettaient en abyme le geste de les traduire et montraient la marche à suivre. Nuit blanche en Balkhyrie présente ainsi un exemple remarquable de minoration d'une langue par appauvrissement, dans l'usage que font les révolutionnaires du balkhyr. On comprend que le monde est totalitaire en ce qu'une seule langue y est en vigueur, le balkhyr ; or les insurgés du monde concentrationnaire qui font la révolution dans ce monde se limitent à "marmonn[er] quelques éléments de conversation courante, des banalités tirées d'une méthode de balkhyr ${ }^{10}$, sans intention de communiquer : "[bien] que faisant l'effort de m'exprimer en sa langue, précise le narrateur Breughel, je n'avais pas l'intention d'entrer en communication avec [la gardienne de camp] » $(N B B, 37)$. Alors même qu'ils en apprennent les rudiments, ils ruinent cette langue entropique aussi sûrement que l'armée de leur généralissime Kirghyl dévaste la terre balkhyre.

Il s'agit bien de destruction, comme chez ce dramaturge de Novarina qui «ne cherch[e] pas à dominer le français, le posséder, mais au contraire à l'empirer, à le mener à sa fin ${ }^{11}$; mais c'est que l'achèvement de tout est un appel, que ce désastre des langues est résurrectionnel. La loi de la révolution est dialectique, en effet : Zamiatine la qualifie bien de mortelle, comme je l'ai noté, mais de préciser aussitôt que «cette mort est destinée à

\footnotetext{
${ }^{9}$ DELEUZE, Gilles, GUATTARI, Félix. Kafka : Pour une littérature mineure. Paris : Editions de Minuit, 1975. Coll. Critique, p. 35.

${ }^{10}$ VOLODINE, A. Nuit blanche en Balkhyrie. Paris : Gallimard, 1997. Coll. Blanche, p. 33. Référence abrégée : $N B B$.

${ }^{11}$ NOVARINA, Valère. Le Théâtre des paroles. Paris : P.O.L, 1989. Entrée dans le théâtre des oreilles, p. 67.
} 
concevoir une vie nouvelle, un astre $»^{12}$. Ainsi Novarina peut-il produire une pièce de théâtre "en français crépusculaire, en langue naissante ${ }^{13}$ : comme si le drame de cette pièce était la mort d'une langue nationale et sa régénération comme langue indistincte quant à sa nationalité. C'est bien ce qui se joue dans la poésie de Celan, s'il est vrai qu'écrire dans une contre-langue, cela revient là, contre le délire nazi de purification, à «rejudaïser » l'allemand ${ }^{14}$, et même à "[faire] de l'allemand une toile de fond, une sorte de palimpseste [que Celan] ne cessera jamais d'enrichir par l'apport de mots, de nuances et d'atmosphères issus d'autres contextes culturels $»^{15}$. Et quant à la littérature post-exotique, l'exemple de l'usage révolutionnaire du balkhyr - spécialement par un narrateur décérébré à la suite d'une lobotomie - montre qu'il s'agit de faire subir aux langues entropiques une cure d'idiotie, de remonter dans le temps des langues jusqu'à leur stade prénatal, - là où n'importe quel mot contient toutes les langues, où toutes les langues sont rendues à leurs possibles inaccomplis (gelés depuis le début de leur évolution nationale), où elles forment toutes ensemble «un immense territoire international $»^{2}$.

Que seule demeure "la langue trans-nationale des conteurs d'histoires, des exclus, des prisonniers, des fous et des morts $»^{2}$, tel est le mot d'ordre utopique pour un recommencement, en effet. Ceux qui, dans Le Nom des singes, veulent remonter en pirogue l'Abacau pour «fonder un territoire utopique ${ }^{16}$ ne disent pas autre chose, quand ils projettent qu' "[en] tant que langue des plus démunis, le cocambo deviendrait langue officielle » $(N S, 176)$; et dans un discours aux araignées sur la révolution, l'un d'entre eux insiste encore, à la fin du livre, sur cet idéal de pauvreté : "niveler au plus bas pour commencer... balbutie-t-il [...] parler la langue des oubliés... celle de ceux qui ont été tués... de ceux qui n'existent plus...» (NS, 239).

Cependant, ce dernier discours a lieu, de façon tragique et dérisoire, en pleine débâcle : le voyage vers l'amont du fleuve des langues, vers un avant de leur AB(a)C(au), a

\footnotetext{
${ }^{12}$ ZAMIATINE, E. Op. cit., p. 151.

${ }^{13}$ NOVARINA, V. Op. cit. Entrée dans le théâtre des oreilles, p. 77.

${ }^{14}$ Cf. ERTEL, Rachel. Brasier de mots. Paris : Editions Liana Levi, 2003, p. 212. Ertel note que Celan fait "éclater dans ses poèmes écrits en allemand, dans la langue des bourreaux, ces vocables étrangers [yiddish], comme autant d'entailles, kaddish, yizkor, gebentcht, havdalah, ou ces vers d'une chanson yiddish [...] ».

${ }^{15}$ TRAVERSO, Enzo. L'Histoire déchirée. Essai sur Auschwitz et les intellectuels. Paris : Les Editions du Cerf, 1997. Paul Celan et la poésie de la destruction [article en ligne s. p.]. Disponible sur: http://www.antirev.org/textes/Traverso97a6/index.html. (Consulté le 05.09.05.)

${ }^{16}$ VOLODINE, A. Le Nom des singes. Paris : Editions de Minuit, 1994, p. 49. Référence abrégée : NS.
} 
tourné court ; les personnages survivants finissent leur route, agonisants, en face de leur point de départ, seulement transportés sur l'autre rive.

A l'instar du théâtre de Novarina, les romans de Volodine viennent "rappeler que parler est un drame ${ }^{17}$. Si les personnages «ont encore le courage de parler, alors qu'ils ont aussi perdu la bataille contre le silence $»^{2}$, c'est pour éprouver que «[s]'exprimer n'aide pas à vivre. [Que les] mots, comme le reste, détruisent ${ }^{18}$. Même armé de sa contre-langue révolutionnaire, on ne met pas un cap vers des langues entropiques «enrichies » de la mort de la révolution sans pâtir de ce voyage. Ce n'est pas seulement que cette catastrophe, charriée par les mots, revienne : si, au bout du compte, toujours, la fiction tourne au cauchemar, c'est que cette catastrophe n'a jamais cessé d'advenir, est éprouvée de l'intérieur par ceux qui parlent.

La révolution est morte dans le monde, mais elle est encore en train de mourir dans le monde concentrationnaire. Et c'est le lot des personnages du post-exotisme, qui sont les derniers révolutionnaires, que de vivre dans leur chair les jours de cette mort. Ils prennent alors la parole pour contredire cette chronique d'une mort annoncée, mais, tandis que leur langue transnationale se voue à lutter contre l'entropie des langues ennemies, elle ne peut être qu'elle-même affectée par l'entropie.

Pour une question de réception, d'abord : parce que si la révolution est morte dans le monde, il en est de même de la langue de la révolution. Et devoir produire un document de culture révolutionnaire dans une langue dont on est le dernier locuteur ou presque et que quasiment plus personne ne peut donc entendre, voilà qui pourrait pour le moins faire douter de la peine qu'on se donne de sauver cette culture. Seule la solidarité inconditionnelle unissant les uns aux autres les personnages du post-exotisme peut leur éviter de sombrer dans le désespoir et de basculer dans la folie, face à une telle extrémité ; c'est bien ce qui ressort de leur renfermement sur soi : ils ne se reconnaissent bientôt plus guère d'autres destinataires qu'eux-mêmes, lecteurs en réclusion, vivants ou morts ( $c f . P E, 43$ ).

Cependant, il n'est pas besoin de circonstances particulières, dans le monde concentrationnaire, pour que la folie menace les hommes et les femmes qu'on y a relégués à perpétuité, puisque c'est la raison d'être du camp de concentration que de conduire les êtres à toucher le fond, de détruire leur psyché. "[Nous] devons prendre conscience du fait que la

\footnotetext{
${ }^{17}$ NOVARINA, V. Pendant la matière. Paris : POL, 1991. Fragment XII, p. 9.

${ }^{18}$ VOLODINE, A. Le Port intérieur. Paris : Editions de Minuit, 1995, p. 9. Cette leçon (ici placée à l'incipit) est une réminiscence célinienne. $C f$. CELINE, Louis-Ferdinand. Voyage au bout de la nuit. Paris : Gallimard, 1972. Coll. Folio, p. 615. "On ne se méfie pas d'eux les mots et le malheur arrive », résume Bardamu au sujet de son voyage.
} 
psyché peut être détruite sans que l'homme soit, pour autant, physiquement détruit, avertissait Hannah Arendt ; que, dans certaines circonstances, la psyché, le caractère et l'individualité ne semblent assurément se manifester que par la rapidité ou la lenteur avec lesquelles ils se désintègrent. " ${ }^{19} \mathrm{C}$ 'est l'expérience centrale du monde concentrationnaire : celle de ces êtres qu'à Auschwitz, on a appelé les Muselmänner (les «musulmans»), et pour qui Primo Levi témoigne, justement parce que ces "non-hommes en qui l'étincelle divine s'est éteinte », qu' " [on] hésite à appeler des vivants », sont « le nerf du camp ${ }^{20}$.

La violence que subissent les personnages du post-exotisme est de cet ordre, celle d'un état d'exception où l'on se sent immédiatement " contesté comme homme, comme membre de l'espèce ${ }^{21}$, où, malgré qu'on en ait, l'on sent affleurer en soi de toutes parts le «musulman ». On doit prêter attention au fait que, quand Volodine parle «des peurs de [ses] narrateurs [qu'il peut] partager avec eux», il n'évoque pas la mort directement, mais «la perte de l'inconscient » : "l'inconscient qui est perdu lorsqu'on meurt, mais qui est également presque perdu quand on est fou $»^{22}$. La mort, dans les camps, arrive en effet alors qu'on est trop épuisé pour la comprendre, elle n'est plus qu'un épiphénomène par rapport à ce qu'il a fallu endurer auparavant, qui est bien plus effrayant : "la mort non-mort et la vie non-vie » $(P E, 75)$; et ce qui se substitue à l'angoisse de la mort dans de telles circonstances, c'est alors l'angoisse de ne pas vraiment mourir, de rester pris indéfiniment dans un état de non-vie et de non-mort.

Si la langue de la révolution est affectée par l'entropie, c'est donc aussi une question d'émission. Car sentir monter en soi le non-homme, être le témoin de sa propre débâcle, de sa perte comme sujet, c'est fondamentalement l'expérience d'une perte du langage. Parler est une défaite contre le silence qui s'ajoute à toutes les batailles perdues par les personnages du post-exotisme, nous dit Volodine, mais on peut même avancer que c'est une défaite qui représente toutes les autres, de façon performative, dans son mode d'être. On voit bien, quand la fiction tourne au cauchemar, que leurs défaites sont exprimées par ce que disent les narrateurs; mais il faut encore considérer que leurs défaites se communiquent en ce qu'ils disent, dans leur manière de signifier $^{23}$. Et c'est ce qui rend la lecture des textes post-

\footnotetext{
${ }^{19}$ ARENDT, Hannah. Les Origines du totalitarisme : 3. Le Système totalitaire. Trad. de l'américain par J.-L. Bourget, R. Davreu et P. Lévy. Paris : Le Seuil, 1972. Coll. Points Essais, p. 177.

${ }^{20}$ LEVI, Primo. Si c'est un homme. Trad. de l'italien par Martine Schruoffeneger. Paris : Pocket, 2003. Coll. Presses Pocket, p. 138-139.

${ }^{21}$ ANTELME, Robert. L'Espèce humaine. Paris : Gallimard, 1978. Coll. Tel, p. 11.

${ }^{22}$ SAVARY, Philippe. «L'écriture, une posture militante ». Le Matricule des anges [en ligne], juillet-août 1997, $\mathrm{n}^{\circ}$ 20. Disponible sur : http://www.lmda.net/mat/MAT02031.html [s. p.]. (Consulté le 31.07.2004.)

${ }^{23}$ Cf. BENJAMIN, Walter. CEuvres. T. I. Trad. de l'allemand par M. de Gandillac, R. Rochlitz et P. Rusch. Paris : Gallimard, 2000. Coll. Folio/Essais. Sur le langage en général et sur le langage humain, p. 143. Benjamin
} 
exotiques souvent si poignante, car leur espace-temps est celui du mourir, de l'agonie, de la survie; or tandis que le psychisme des narrateurs se désintègre, leur langue se disloque, se désagrège.

Le parallèle entre la littérature post-exotique et la poésie de Paul Celan s'impose encore ici. La façon dont la Shoah se communique dans l'œuvre du poète de langue allemande a paru insoutenable à Primo Levi :

Ces ténèbres, de plus en plus denses de page en page, jusqu'à un ultime balbutiement inarticulé, consternent comme le râle d'un moribond, et le sont en effet. Elles nous attirent comme attirent les gouffres, mais en même temps elles nous flouent de quelque chose qui devait être dit et ne l'a pas été, et donc elles nous frustrent et nous repoussent. Je pense, quant à moi, que le poète Celan doit bien plutôt être médité et pris en compassion qu'imité. Si son message est un message, celui-ci se perd dans le "bruit": il n'est pas une communication, il n'est pas un langage, tout au plus est-il un langage obscur et manchot, tel celui de qui va mourir, seul comme nous le serons tous à l'agonie. ${ }^{24}$

Et la façon dont la mort des derniers révolutionnaires se communique dans la littérature postexotique consterne également. La leçon onze du Post-exotisme en dix leçons, leçon onze narre ainsi la fin de Lutz Bassmann, ses derniers jours dans le quartier de haute sécurité d'une prison. Le texte évoque "ses derniers soubresauts romanesques [qui] s'abritent sous des titres peu attirants et peu travaillés» $(P E, 17)$, d'ultimes romånces dont «le ressassement [...] ne repose plus sur rien de communicable. [...] On a là les borborygmes terminaux, les ultimes rauqueries scandées du post-exotisme... » $(P E, 18)$. Mais le texte ne fait pas que les évoquer : le texte est un dernier soubresaut romanesque, son rythme est l'effort de survie que produit Bassmann :

Mot à mot, râle après râle, Lutz Bassmann se battait pour faire durer l'édifice mental qui allait redevenir poussière. Son haleine se confondait avec les sueurs putrides qui erraient dans la prison. Il s'accrochait encore un peu au réel et il s'arrangeait pour que les débris. Il s'arrangeait pour que sa voix ne s'évanouisse pas encore. Pour qu'une heure de plus, deux heures et demie, une nuit de plus, persistent les mondes que nous avions tenu à charpenter âprement et à défendre. Edifice mental... Monde... Âpre charpente... Qu'est-ce que... Hein ?... (PE, 16-17.)

fait constamment la distinction entre «ce que par [le langage] nous croyons pouvoir exprimer [et] ce qui en lui se communique. ». Cf. Ibid. La tâche du traducteur. Benjamin souligne également qu'il faut «distinguer ce qui est visé de la manière dont on le vise » (p. 251), car le sens "ne s'épuise pas dans ce qui est visé, mais acquiert justement cette signification par la manière dont ce qui est visé est lié, dans le mot déterminé, au mode de la visée » (p. 256).

${ }^{24}$ LEVI, P. Le Métier des autres. Cité par AGAMBEN, Giorgio. Ce qui reste d'Auschwitz : L'archive et le témoin: Homo Sacer III. Trad. de l'italien par Pierre Alferi. Paris : Editions Payot \& Rivages, 2003. Coll. Rivages poche / Petite bibliothèque, p. 39. 
Dans cet instant du récit où Bassmann est prêt de sombrer dans l'inconscience, la langue bégaie, s'essouffle, se suspend, les phrases se brisent. Puis, dans un sursaut ("Qu'est-ce que... Hein ?... »), le personnage retrouve quelque peu ses esprits et la syntaxe, alors, dans un filet de voix, un souffle à peine audible, reprend en partie ses droits, jusqu'à la prochaine fois ; et ainsi de suite, jusqu'à ce que mort s'ensuive :

La prison immense gémissait comme un bateau abandonné par les rats, par l'équipage, et qui attendait encore un peu avant de s'abîmer. Dans les couloirs, le vent sifflait, et parfois, prophétisant la dernière phrase sur quoi s'arrêterait le souffle post-exotique, il se taisait. On avait du mal à établir une frontière entre les bruits d'eau émis par le déluge, les râles de Bassmann émis par Bassmann, les simulacres de souvenirs émis par les photographies de ceux qui avaient été nos surnarrateurs. Le post-exotisme s'achevait là. La cellule sentait le monde décomposé, l'humus brûlant, la fièvre terminale, elle empestait les peurs que les animaux les plus humbles, et je le regrette, ne trouvent jamais les mots pour dire. Il n'y avait plus un seul porte-parole qui pût succéder à. C'est donc moi qui $(P E, 85$.)

Les râles de Bassmann dévasté par la fièvre se perdent dans le bruit (comme le message de Celan suivant Levi), on est privé de ses derniers mots, et le texte s'interrompt brusquement sur un balbutiement, plutôt qu'il ne s'achève, avec sa mort.

Dans la littérature post-exotique comme dans la poésie de Paul Celan - malgré Primo Levi -, on trouve un état de la langue où elle tend à se confondre avec le chant disharmonieux de l'oiseau dans le crépuscule, qui «n'est même plus de l'épouvante, seulement la dernière ruine sonore avant la non-lumière, seulement un sursaut mécanique dans quoi peur et nonpeur sont devenues abstraites et s'équivalent » $(N B B, 36)$, et ce qu'explicite même la fin du Post-exotisme en dix leçons, leçon onze, c'est que cet état de la langue est son horizon : une «longue série de kwaks et de kiïkiïks monotones » $(N B B, 36) \ldots$ puis la nuit.

La fin tragique du Post-exotisme en dix leçons, leçon onze sonne le glas du postexotisme ; la défaite est consommée. Si ce n'est qu'une œuvre reste, comme « une bouteille à la mer, mise à l'eau dans la croyance - pas toujours forte d'espérances, certes - qu'elle pourrait être en quelque lieu et quelque temps entraînée vers une terre, Terre-Cour peut$\hat{e} t r e »^{25}$. Parler est plus qu'une ultime défaite qui représente toutes les défaites passées, si cette

\footnotetext{
${ }^{25}$ CELAN, P. Op. cit. Allocution de Brême, p. 57. Paul Celan a été le traducteur d'Ossip Mandelstam, et il lui emprunte ici l'image du message dans une bouteille jetée à la mer ; cette image prend une résonance poignante si l'on se souvient que, de Mandelstam englouti dans les camps de Staline en 1938, maints poèmes nous sont parvenus de façon inouïe, grâce à la mémoire fervente de sa femme, Nadejda (« espérance », en russe), qui les a appris par cœur et cachés au fond d'elle-même pour les soustraire aux investigations de la police politique soviétique.
} 
ultime défaite produit une œuvre restante, car ce reste, c'est ce qui peut transformer la défaite en son contraire, en dernier ressort.

C'est une possibilité qui tient à la tension testimoniale de la parole post-exotique. On peut reconnaître en effet qu'il y a dans le narrateur post-exotique deux postulations simultanées: l'une vers le «musulman», l'autre vers le rescapé. C'est un drame qui commence dès la prise de parole, parce que le personnage, que le monde concentrationnaire entraîne dans un mouvement de désubjectivation, sent immédiatement que sa parole risque de « se métamorphoser en désordre bavard» $(N B B, 14)$; néanmoins, la langue étant la matière dont l'homme est fait, "l'homme [n'étant] pas dans un monde, mais dans une langue, jeté $»^{26}$, c'est dans une langue, qui seule lui reste, que le personnage pense pouvoir défendre l'homme contre le non-homme, et il tente d'initier un mouvement de resubjectivation dans une littérature.

Dans un sens, l'homme perd cette bataille, j'en ai rendu compte. Dans la langue du narrateur post-exotique, dans l'écriture poétique de sa langue, il n'y a bientôt plus que du schibboleth. Qu'on considère l'action destructrice de la langue transnationale post-exotique sur les langues entropiques ou, inversement, l'action destructrice de l'entropie concentrationnaire sur la langue transnationale, c'est un même phénomène qui s'observe : le sens chute peu à peu dans l'abîme de la langue, entraînant le narrateur dans la folie et le néant, jusqu'à "l'aporie d'une absolue désubjectivation et "barbarisation" de l'événement langagier, où le sujet parlant cède la place à un autre, enfant, ange, barbare, qui parle "en l'air" et de façon "stérile" ${ }^{27}$, comme dans les dernières œuvres logorrhéiques de Lutz Bassmann, dont «[le] cryptage est vain, [l']indéniable beauté est vaine » $(P E, 18)$ : car alors, la langue est un reste qui survit à une incapacité de parler.

Mais dans un autre sens, le narrateur post-exotique produit une œuvre de langage, et celle-ci, dialogique par essence, peut faire que l'homme survive au non-homme, en définitive : parce qu'entre l'écrivain et le lecteur, «en lisant, en écrivant, il se produit de l'homme, il naît de l'homme en parlant ${ }^{28}$.

Ce n'est pas seulement qu'un genre post-exotique, par exemple le romånce, "introduit en lui, en tant que composante importante de la fiction, une représentation de son lecteur », lequel est toujours «un des personnages du post-exotisme» $(P E, 42-43)$. La littérature post-

\footnotetext{
${ }^{26}$ NOVARINA, V. Le Théâtre des paroles. Entrée dans le théâtre des oreilles, p. 79-80.

${ }^{27}$ AGAMBEN, G. Op. cit., p. 125. Quant au danger que représente l'action de faire perdre le sens aux langues entropiques avec sa langue transnationale, on peut se reporter à l'analyse par Walter Benjamin de ce qui a causé la folie de Hölderlin : $c f$. BENJAMIN, W. Op. cit. La tâche du traducteur, p. 261.

${ }^{28}$ NOVARINA, V. Le Théâtre des paroles. Chaos, p. 154.
} 
exotique s'écrit bien pour un personnage du post-exotisme, mais il ne faut pas entendre pour qu'au sens d' « à destination de » : une singularité fondamentale de cette littérature consiste en ce qu'un écrivain conçoit toujours un texte «à la place » d'un autre, et spécialement d'un camarade disparu ; ainsi en est-il dans le romånce, où le surnarrateur post-exotique, « en un processus de camaraderie intime, contraint sa voix et sa pensée à reproduire la courbe mélodique d'une voix et d'une pensée disparues » $(P E, 39)$.

Or cette solidarité post-exotique appelle un commentaire déterminant, pour ce qui nous occupe. C'est d'abord que la désubjectivation qu'induit le monde concentrationnaire est prise en charge par une entreprise littéraire où "il n'y a pas l'épaisseur d'une feuille de papier à cigarette entre la première personne et les autres » $(P E, 19)$, où « [les] personnalités s'échang[ent], les signatures se mêl[ent], comme dans l'amitié on mêle les sangs » (PE, 58). S'il est vrai que l'écrivain est toujours étranger à lui-même dans le temps opératoire de l'écriture, cette vérité littéraire est ici érigée en principe, dans la mesure où s'instaure tout un système hétéronymique suivant lequel les narrateurs, qui parlent à la première personne, sont en fait - comme les «musulmans », qui sont sans visages, une masse anonyme - toujours interchangeables, substituables les uns aux autres, de «fraternelles variantes » $(P E, 70)$ les uns des autres. Cependant - et c'est la seconde partie du commentaire -, cela ne revient pas à parler de résignation au régime de désubjectivation du camp. Au contraire, c'est comme si le régime du camp était instrumentalisé et détourné, car ce système hétéronymique a pour corollaire de favoriser un processus de resubjectivation collective, dans ce que les narrateurs appellent parfois un «moi insoluble» ( $c f . N B B, 23 ; P E, 70)$. Et la beauté de ce dispositif d'écriture, c'est qu'il applique un principe qui guide désormais leur action de révolutionnaires survivants, à savoir le «partage radical de la douleur et du malheur entre tous » $(N B B, 130)$; ils livrent leur dernière bataille dans le langage «agglutinés os à os pour le malheur et pour le pire $(N B B, 124)$, et sous cette forme se communique l'utopie égalitariste qu'ils ont eu la force de ne jamais renier.

Le narrateur post-exotique devient le sujet collectif d'un témoignage en tant qu'il témoigne d'une désubjectivation collective ${ }^{29}$ et c'est en cela qu'il tend vers le rescapé. La désubjectivation concentrationnaire participe du processus par lequel il fait corps avec ses camarades dans une littérature, et celle-ci rassemblant ainsi une commune égalitariste au sein du monde concentrationnaire, elle devient un territoire d'exil intérieur $(c f . P E, 36)$; or faire

\footnotetext{
${ }^{29}$ Ma réflexion sur le témoignage s'inspire de Ce qui reste d'Auschwitz, d'Agamben : cf. Op. cit., p. 130-132.
} 
retraite de cette manière communiste dans le monde concentrationnaire crée une ligne de fuite vers son dehors, lance comme un appel à une communauté à venir, ailleurs.

Cependant - c'est un écueil dont un aspect a été entrevu -, pour que cet appel soit entendu, encore faut-il que les bouteilles à la mer post-exotiques puissent atteindre une terre hors $\mathrm{du}$ monde concentrationnaire: qu'elles ne soient pas elles-mêmes purement et simplement détruites comme on élimine les hommes et femmes qui les fabriquent, et si elles tombent entre des mains amies, qu'on puisse les déchiffrer.

On imagine bien qu'un pouvoir totalitaire qui a entrepris de détruire totalement une culture n'est nullement disposé à laisser lui échapper des livres dont le cryptage fait déjà l'objet d'interrogatoires sans fin à l'intérieur du monde concentrationnaire. Tout laisse penser que le devenir de la littérature post-exotique ne peut être que l'histoire d'une destruction ; que l'image d'un "livre traîn[ant] dans les déjections et le sang ${ }^{30}$ qui ouvre le premier opus post-exotique connu résume cette histoire tout entière. Et si l'on se reporte à la bibliographie du post-exotisme que présente la leçon dix du Post-exotisme en dix leçons, leçon onze, on a la sensation désagréable, en effet, de découvrir le catalogue d'une bibliothèque engloutie. Il s'en faut de peu qu'on ne distingue rien qui ait été jamais édité, parmi les 343 titres recensés ; seul un examen attentif permet de repérer notamment dix titres identifiables, pour les années 1977 à 1998 :

[...] / 44 BIOGRAPHIE COMPARÉE DE JORIAN MURGRAVE, romånce, attribué à Iakoub Khadjbakiro, 1985 [...] / 54 UN NAVIRE DE NULLE PART, romånce, attribué à Wernieri, 1986 [...] / 57 RITUEL DU MÉPRIS, romånce, attribué à Julio Sternhagen, 1986 [...] / 73 DES ENFERS FABULEUX, romånce, attribué à Lilith Schwack, 1988 [...] / 86 LISBONNE, DERNIÈRE MARGE, romånce, attribué à Vassilissa Lukaszczyk, 1990 [...] / 91 ALTO SOLO, romånce, attribué à Jean Vlassenko, 1991 [...] / 130 LE NOM DES SINGES, romånce, attribué à Yasar Tarchalski, 1994 [...] / 182 LE PORT INTÉRIEUR, romånce, attribué à Rita Hoo, 1996 [...] / 217 NUIT BLANCHE EN BALKHYRIE, romånce, attribué à Aidan Sherrad, 1997 [...] / 226 VUE SUR L'OSSUAIRE, romånce, attribué à Jean Khorassan $[\ldots](P E, 88-100$.

Ces titres, on peut le vérifier, sont parus en français sous la signature d'Antoine Volodine à des dates qui concordent ; mais leur présence dans cette bibliographie au milieu d'autres titres inconnus, leur attribution incertaine à des auteurs tout aussi inconnus, - voilà qui leur confère une dimension lazaréenne, car il semble bien que sans Volodine, ces titres auraient disparu comme les autres.

\footnotetext{
${ }^{30}$ VOLODINE, A. Biographie comparée de Jorian Murgrave, Un Navire de nulle part, Rituel du mépris, Des Enfers fabuleux. Paris : Denoël, 2003. Coll. Des heures durant... Biographie comparée de Jorian Murgrave, p. 17.
} 
Dès lors qu'on comprend que Volodine fait œuvre de traducteur, on mesure ce que sa tâche, bien que mineure, a d'exceptionnel. Non seulement il sauve des œuvres de la destruction physique alors que, dans le monde concentrationnaire, à l'état de samizdats, en très peu d'exemplaires ${ }^{31}$, elles paraissaient à la merci du pouvoir totalitaire (mais on fait effectivement mention, dans le Post-exotisme en dix leçons, leçon onze, de « romånces dont plusieurs volumes avaient paru à l'extérieur de la prison, sous la signature d'un [...] prête$n o m »(P E, 19))$; non seulement il évite à ces œuvres d'être détruites, donc, mais s'il prend la peine de les traduire, c'est encore pour leur éviter d'être illisibles, puisque la langue transnationale post-exotique est morte hors du monde concentrationnaire.

Il y a là comme un passage de témoin. En premier lieu, en ce que Volodine répond à l'appel d'une langue qui est pure traductibilité. Il ne peut le faire que parce qu'il est intimement lié à la communauté qui la parle, que cette langue transnationale est la sienne ; et sa fidélité aux siens consiste alors à, "amoureusement et jusque dans le moindre détail, adopter dans [la langue d'arrivée] le mode de visée de l'original, afin de rendre l'un et l'autre reconnaissables comme fragments d'un même vase ${ }^{32}$. C'est faire la révolution dans le français : le minorer, en l'appauvrissant ou en l'exténuant, l'élargir et l'approfondir au moyen de la langue transnationale, le soumettre à l'action de celle-ci contre l'entropie, se faire l'écho de celle-ci de sorte que le français sonne comme une langue étrangère indistincte, - de sorte qu'on y perde « son » français.

En ce sens, déjà, on peut dire que la traduction de Volodine procède de la survie de l'original post-exotique, qu'elle est une survivance de sa culture révolutionnaire. Mais cette réflexion prend évidemment une résonance particulière dans le contexte de la condamnation à mort décrétée contre cet original, contre cette culture. C'est pourquoi on peut ici parler de posture de témoin du traducteur, de valeur de témoignage de sa traduction. Car se faire l'écho de la langue transnationale post-exotique, ce n'est pas seulement s'attaquer à l'entropie à sa manière, c'est aussi, inversement, témoigner de ce qu'elle a été elle-même attaquée par l'entropie, et ce au point d'en mourir, ou presque.

Traduire une langue en voie d'anéantissement, c'est une manière de commencer un travail de deuil, car c'est témoigner qu'elle a été et qu'elle n'est bientôt plus, " c'est tenter d'inscrire dans la langue de traduction la [quasi-]mort de la langue de départ ${ }^{33}$. Or,

\footnotetext{
${ }^{31}$ Cf. VOlODINE, A. Dondog. Paris : Le Seuil, 2002. Coll. Fiction \& Cie, p. 106. «Pendant mes loisirs, raconte Dondog, [...] j'écrivais des livres pour le public du camp, de petits ouvrages dont circulait un nombre d'exemplaires parfois non ridicule, quoique jamais supérieur à cinq ou six. »

${ }^{32}$ BENJAMIN, W. Op. cit. La tâche du traducteur, p. 257.

${ }^{33}$ ERTEL, R. Op. cit., p. 245.
} 
comme la langue transnationale post-exotique se meurt en ses derniers locuteurs, la tâche du traducteur implique de se confronter à l'impossibilité quasi physique de parler des narrateurs post-exotiques, à leur suffocation qui se communique dans la langue, à cette langue barbare qui parle toute seule au-delà de leur absolue désubjectivation; et c'est «aller chercher, dans le naufrage du siècle, des éclats, des lambeaux, des fragments, des gravats, des "motsépaves" » ${ }^{34}$ : tout ce dont le français s'est « enrichi », qui peut faire entendre cette abolition du sens dans la langue de départ, qui peut rendre compte de sa ruine.

Survivance de l'original post-exotique et de sa culture révolutionnaire, la traduction l'est encore en tant que témoignage ; cependant, si, telle un testament, elle implique et confirme la mort de cet original, de cette culture, elle commence une vie après cette mort, impliquant et confirmant alors la possibilité de résurrection qu'ils appelaient ; et elle offre la communauté post-exotique en héritage, le schibboleth étant «la clef qui [donne] accès à une "communauté", à un "nous" »".

\footnotetext{
${ }^{34}$ Ibid., p. 188.

${ }^{35}$ Ibid., p. 219.
} 